\title{
Sintomatologia de depressão autorreferida por idosos que vivem em comunidade
}

\author{
The symptomatology of self-referred depression \\ by elderly people who live in a shantytown
}

Marcos Francisco de Oliveira ${ }^{1}$

Valéria Peixoto Bezerra ${ }^{2}$

Antonia Oliveira Silva ${ }^{2}$

Maria do Socorro Costa Feitosa Alves ${ }^{3}$

Maria Adelaide Silva Paredes Moreira ${ }^{4}$

Célia Pereira Caldas ${ }^{5}$

${ }^{1}$ Secretaria de Saúde de Campina Grande (PB).

Manoel Alves de Oliveira no 793/101 B, Catolé. 58410575 Campina Grande PB. marcosfcco@gmail.com

${ }^{2}$ Programa de PósGraduação em Enfermagem, Departamento de Enfermagem Clínica, Universidade Federal da Paraíba.

${ }^{3}$ Programa de PósGraduação em Saúde Coletiva, Universidade Federal do Rio Grande do

Norte.

${ }^{4}$ Programa de Pós-

Graduação em Enfermagem e Saúde, Universidade Estadual do Sudoeste da Bahia.

${ }^{5}$ Programa de PósGraduação em Enfermagem, Universidade do Estado do Rio.

\begin{abstract}
The scope of this quantitative exploratory study was to evaluate the symptomatology of self-referred depression by the elderly, taking socio-demographic variables into consideration. The data collected by means of semi-structured interviews and the Abbreviated Geriatric Depression Scale involving 240 elderly residents in João Pessoa in the state of Paraiba between October and December 2010 were analyzed by Excel and SPSS software. It was found that $75.8 \%$ had no degree of symptomatology of depression, and $24.2 \%$ manifested mild or severe depression. According to these variables, in the elderly patients with depression it was found that: in relation to age, the elderly with mild and severe depression appear more frequently between 71 to 76 years $-31 \%$; in relation to gender, females stand out with $86 \%$; on marital state, married couples with $41.3 \%$, and widowers with $34.5 \%$; considering family income, from 1 up to 3 minimum wages, $50 \%$, revealed mild incidence; with respect to schooling, elderly people who have no depression, $84.6 \%$, can read and write. Therefore, it can be said that the symptomatology of depression in the elderly is related to socio-economic and cultural conditions, emphasizing the need for public health policies that ensure comprehensive care and quality considering these variables.
\end{abstract}

Key words The elderly, Depression, Health, Disease
Resumo Este estudo exploratório quantitativo objetivou avaliar a sintomatologia da depressão autorreferida por idosos, considerando variáveis sociodemográficas. Os dados coletados por meio de entrevista semiestruturada e da Escala de Depressão Geriátrica Abreviada envolvendo 240 idosos residentes em João Pessoa (PB), no período de outubro a dezembro de 2010, foram analisados pelos softwares Excel e SPSS. Verificou-se que 75,8\% não apresentaram grau de sintomatologia de depressão e 24,2\% manifestaram depressão leve ou severa. De acordo com essas varáveis, nesses idosos com grau depressivo verificou-se que: em relação à faixa etária, aqueles com grau depressivo leve e severo destacam maior frequência entre 71 a 76 anos, 31,0\%; com relação ao gênero, o feminino destaca-se com 86,0\%; sobre o estado civil, os casados com $41,3 \%$ e os viúvos com $34,5 \%$; considerandose a renda familiar, os de 01 até 03 salários mínimos, 50\%, apresentaram grau leve; com vistas à escolaridade, dos que não evidenciaram grau de depressão, 84,6\%, sabem ler e escrever. Portanto, pode afirmar-se que a sintomatologia da depressão em idosos apresenta relação com as condiçães socioeconômicas e culturais, fortalecendo a necessidade de políticas públicas de saúde que garantam um atendimento integral e de qualidade, considerando essas variáveis.

Palavras-chave Idoso, Depressão, Saúde, Doença 


\section{Introdução}

O aumento da longevidade da população mundial é, sem dúvida, um valioso trunfo alcançado pela humanidade. A Organização Mundial da Saúde, OMS, preocupada com a qualidade de vida das pessoas na fase idosa, recomenda aos países desenvolvidos e em desenvolvimento que busquem a promoção de um envelhecimento voltado para a manutenção da atividade funcional e da autonomia, acompanhado da melhoria ou manutenção da saúde e da qualidade de vida ${ }^{1}$.

O envelhecimento é um processo em que, no indivíduo, ocorrem mudanças morfológicas, funcionais e bioquímicas, que alteram progressivamente o organismo ${ }^{2}$. Contudo, é importante ressaltar que esse processo acontece de modo diferente para cada indivíduo. Sendo assim, é necessário que os profissionais da saúde tenham uma visão holística do idoso.

Ao chegar a essa fase, é provável que a pessoa tenha adquirido um acúmulo de experiências que podem levar a um enriquecimento de conhecimentos sobre o modo de viver, embora se considere que não se obteve apenas ganhos e realizações, mas também pode ter havido perdas e/ou limitações. As circunstâncias históricas e socioculturais, a presença de patologias, de fatores genéticos e ambientais são aspectos que possivelmente vão estabelecer a forma como o indivíduo idoso chegou a essa etapa da vida.

A Organização Mundial da Saúde considera que para o idoso ter uma vida mais saudável é necessário um envelhecimento integral e ativo. Assim, é importante que tenha um convívio social, boa capacidade mental, participação na sociedade de acordo com suas satisfações, necessidades, desejos e capacidades, etc. A esses indivíduos devem ser disponibilizadas as condições necessárias para proteção, segurança e demais cuidados que forem adequados.

Nesse sentido, o Ministério da Saúde desenvolve o programa Brasil Saudável, o qual envolve uma ação nacional para a elaboração de políticas públicas que gerem modos de viver saudáveis em todas as etapas da vida, favorecendo a prática de atividades físicas habituais e de lazer, o acesso à alimentação saudável, com a redução do consumo de taba$\mathrm{co}^{3}$. Tais ações visam preservar as capacidades funcionais essenciais e a manutenção das atividades diárias, que objetivam manter o idoso na comunidade, próximo da família, evitando a sua transferência para Instituições de Longa Permanência.

No que se refere à saúde, este é um fator extremamente importante na busca pelo envelhe- cimento ativo das pessoas idosas em todo o mundo. Doenças Crônicas Não-transmissíveis (DCNT), tais como a depressão ${ }^{1}$, perfazem um grupo de patologias que podem influenciar negativamente essa etapa da vida.

Considerando que a depressão vem ocorrendo com maior frequência entre as pessoas idosas, configurando-se um relevante problema de Saúde Pública, por essa razão é de fundamental importância que os profissionais de saúde, em especial os que exercem suas funções na Estratégia Saúde da Família (ESF), dispensem maior atenção ao atendimento e acompanhamento desse grupo de pessoas, possibilitando, assim, um diagnóstico precoce, e início imediato do tratamento dessa morbidade.

A depressão é um transtorno psiquiátrico do humor que, desde a antiguidade, tem casos relatados em vários documentos, a exemplo do Primeiro Testamento. No século IV a.C., Hipócrates mencionou os termos "mania e melancolia" quando descreveu os transtornos mentais, afirmando que a etiologia do humor dependia do equilíbrio entre os humores corporais, e que a depressão era causada pelo excesso da bile negra no baço ${ }^{4}$.

Com o avanço tecnológico, os estudos dos humores e sucos melancólicos possibilitaram a descoberta do envolvimento de vários fatores ou causas que podem determinar ou contribuir, significativamente, para o aparecimento da depressã $0^{5}$.

A partir desse período, tem-se observado que a depressão possui aspectos de saúde pública que necessitam do desenvolvimento e da implementação de programas mais específicos para o atendimento do indivíduo de maneira integral, portador de qualquer transtorno do humor, incluindo o deprimido, visando a reinserção deste na sociedade ${ }^{1}$.

Para tanto a elaboração de estratégias e programas é extremamente importante, haja vista que a prevalência e a incidência da depressão têm aumentado significativamente no mundo, principalmente entre os países em desenvolvimento, sendo o Chile o país que apresenta a taxa de incidência mais elevada desta morbidade $(29,0 \%$ da população). No Brasil, a prevalência da depressão varia de $3 \%$ a $11 \%$, na população em geral, com predominância nas mulheres ${ }^{3,6}$.

Na década de 90, essa doença foi considerada a quarta maior causa específica de incapacitação no mundo, e com projeções para 2020 de ser a segunda maior causa nos países desenvolvidos e a primeira nos países em desenvolvimento ${ }^{3}$.

A depressão é caracterizada por uma alteração profunda e duradoura do estado de ânimo 
do indivíduo, persistindo pelo menos por um período de duas semanas e pode afetar o ser humano em qualquer fase da vida - infância, adolescência, adulta e idosa. As pessoas exibem perda de energia e interesse, sentimento de culpa, desempenho social e ocupacional prejudicados, dificuldade de concentração, perda de apetite e pensamentos de morte ou suicídio ${ }^{7}$.

Vários são os fatores que podem determinar ou contribuir para o surgimento da depressão no indivíduo: biológicos, genéticos, psicossociais, distorções cognitivas e impotência apreendida ligada à experiência de acontecimentos incontroláveis ${ }^{4}$.

Para o diagnóstico da depressão, além da observação clínica, os médicos se utilizam de dados genéticos e escala de avaliação. Todavia, outros critérios devem ser analisados durante a avaliação, que são: humor deprimido, diminuição do interesse ou do prazer nas atividades, redução do peso sem dieta, alteração no padrão do sono, agitação ou retardo, fadiga ou falta de energia diária, sentimentos constantes de menos valia ou culpa excessiva ou inadequada, redução da capacidade de concentração ou indecisão, pensamentos recorrentes de morte, ideias e tentativas ou planos de suicídio recorrentes ${ }^{5,8}$.

Nesse aspecto, é imprescindível para a definição diagnóstica dessa morbidade, necessariamente, a presença de, pelos menos, cinco desses critérios, por um período de duas semanas, associada a prejuízo psicossocial ou sofrimento significativo do indivíduo?.

Quanto ao tratamento da depressão, vários regimes farmacológicos, isolados ou associados à psicoterapia, estão sendo utilizados pelos profissionais da saúde. Se não for tratada corretamente, essa doença pode perdurar por 6 a 15 meses. Entretanto, caso a terapêutica seja bemsucedida, o tempo diminui para 8 a 12 meses. Porém, o melhor julgamento clínico para a terapia é uma combinação da farmacoterapia e da psicoterapia, e a escolha vai depender da disponibilidade e da preferência do paciente ${ }^{10}$.

Com relação ao idoso, ao se considerar esses cuidados, o envelhecimento poderá representar um ganho considerável em qualidade de vida e saúde para o indivíduo. Nessa perspectiva, torna-se importante que a saúde seja vista a partir de uma ótica holística e se possível transcendente, resultando em um trabalho intersetorial e transdisciplinar, de promoção de modos de vida saudável, em todas as idades.

Tendo em vista o tema abordado e o universo populacional de idosos, este estudo refere-se à problemática existente em João Pessoa (PB), e tem como objetivo avaliar a sintomatologia da depressão auto referida por tais indivíduos nessa região.

\section{Métodos}

Estudo exploratório, de natureza quantitativa, resulta do Convênio: Universidade Federal da Paraíba (UFPB)/Ministério da Saúde (MS), pelo Projeto - Tecnologias assistivas para idosos atendidos em Unidades de Saúde da Família, como subprojeto: Condições de Saúde, Qualidade de Vida e Representações Sociais de Idosos nas Unidades de Saúde da Família do convênio entre UFPB/ Fundo Nacional de Saúde (FNS/MS), após apreciação e aprovação do Comitê de Ética em Pesquisa do Hospital Universitário Lauro Wanderley (UFPB).

A população foi composta por idosos, residentes em João Pessoa (PB), Brasil, a partir de uma amostra extraída tendo por base a contagem populacional do IBGE, Censo de 2007, de 61.281 indivíduos. Neste estudo, o universo considerado foi de 240 pessoas. Esta amostra foi constituída sorteando-se inicialmente 20 setores censitários, com probabilidade proporcional ao número de domicílios, composto por $617^{11}$, em que se procedeu a sua localização no mapa municipal de João Pessoa (PB), visualizando os bairros correspondentes a cada setor. Para a realização do arrolamento, foram considerados 32 idosos em cada setor, definidos a partir de visitas domiciliares através de sorteio desses setores, em que foram listadas as suas ruas. Em seguida, realizou-se um segundo sorteio, determinando as quadras e ruas a serem visitadas pelo entrevistador. Ao todo, foram arrolados 640 pessoas.

Para a coleta de dados foi utilizado um formulário composto, na primeira parte pelas variáveis: idade, sexo, estado civil, religião, renda e escolaridade; na segunda parte, contemplou-se a Escala de Depressão Geriátrica Abreviada (EDG) ${ }^{3}$. Essa coleta foi realizada no período de outubro a dezembro de 2010, após agendamento de uma visita, de acordo com a disponibilidade dos participantes, atentando para os seguintes critérios de inclusão: a) estar na faixa etária igual aos 60 anos ou mais de idade; b) residir no setor censitário do município, há mais de seis meses; c) possuir condições cognitivas de responder às questões sem ajuda; d) concordarem em participar do estudo, pela assinatura, em duas vias, do Termo de Consentimento Livre e Esclarecido, 
atendendo à Resolução 196/1996, que rege a pesquisa envolvendo seres humanos ${ }^{12}$. No caso do participante não ser alfabetizado, a leitura do referido Termo foi realizado pelo acompanhante.

Os dados foram validados empregando-se dupla entrada (digitação) em banco de dados no aplicativo MS Excel, sendo posteriormente analisados no programa SPSS (Statistical Package for the Social Sciences), versão 11.0. As variáveis categóricas foram analisadas em tabelas de frequência, ao passo que as quantitativas foram descritas com medidas de tendência central e variabilidade. A análise inferencial considerou um nível de significância de 5\%, empregando-se o teste " $\mathrm{t}$ " na análise bivariada e a análise de regressão linear múltipla para a determinação dos preditores mais importantes dos escores de qualidade de vida dos idosos entrevistados.

\section{Resultados e discussão}

Como já mencionado acima, dos 640 idosos arrolados, deste estudo participaram 240 com idade a partir dos 60 anos, conforme Tabela 1 .

Dessa tabela, observa-se que a média de idade dos entrevistados situa-se nos 71,8 anos, sendo o maior número de participantes $(48,4 \%$ idosos) inseridos entre 60 e 70 anos, e uma predominância do sexo feminino com $167(69,4 \%)$ participantes.

Do ponto de vista sociodemográfico, a feminização da velhice pode estar relacionada a fenômenos, tais como a longevidade maior nas mulheres e ao fato delas se cuidarem mais do que os homens e de se exporem menos a riscos, a maior exposição dos homens às doenças cardiovasculares e às taxas elevadas de óbitos por câncer nesse sexo ${ }^{13}$.

Em relação ao estado civil, 52,9\% dos participantes são casados e 33,8\% são viúvos. O fato da maioria ser casada pode contribuir para minimizar os sentimentos de solidão que permeiam essa fase da vida. Esse aspecto pode ser considerado como positivo, pois parece que tal situação contribui para uma menor chance de limitação funcional ${ }^{14}$.

No tocante à religião, verifica-se a partir dos dados da mencionada tabela que 173 (72,1\%) idosos são católicos, $54(22,5 \%)$ protestantes e $12(5,0 \%)$ pertencem a outras religiões. O fato da maioria dos idosos pertencerem à religião católica se justifica em vista do Brasil ser considerado o país com maior número de católicos (61\%), e da Paraíba registrar uma predominância de $84 \%$ da população nessa religião ${ }^{15}$.
Tabela 1. Distribuição percentual das variáveis sociodemográficas dos idosos, João Pessoa (PB), 2011.

\begin{tabular}{|c|c|c|}
\hline \multirow{2}{*}{ Variável } & \multicolumn{2}{|c|}{$\begin{array}{c}\text { Idosos } \\
\text { pesquisados }\end{array}$} \\
\hline & $\mathbf{N}$ & $\%$ \\
\hline \multicolumn{3}{|l|}{ Idade (anos) } \\
\hline 60 a 65 & 58 & 24,2 \\
\hline 66 a 70 & 58 & 24,2 \\
\hline 71 a 76 & 52 & 21,7 \\
\hline 77 a 80 & 41 & 17,1 \\
\hline 81 a 85 & 21 & 8,7 \\
\hline $86 \mathrm{e}+$ & 10 & 4,1 \\
\hline Total & 240 & 100,0 \\
\hline \multicolumn{3}{|l|}{ Sexo } \\
\hline Masculino & 73 & 30,4 \\
\hline Feminino & 167 & 69,6 \\
\hline Total & 240 & 100,0 \\
\hline \multicolumn{3}{|l|}{ Estado civil } \\
\hline Solteiro (a) & 20 & 8,3 \\
\hline Casado (a) & 127 & 52,9 \\
\hline Divorciado (a)/desquitado (a) & 6 & 2,5 \\
\hline Separado (a) & 6 & 2,5 \\
\hline Viúvo (a) & 81 & 33,8 \\
\hline Total & 240 & 100,0 \\
\hline \multicolumn{3}{|l|}{ Religião } \\
\hline Católica & 173 & 72,1 \\
\hline Protestante & 54 & 22,5 \\
\hline Espírita & 1 & 0,4 \\
\hline Outra religião & 12 & 5,0 \\
\hline Total & 240 & 100,0 \\
\hline \multicolumn{3}{|l|}{ Renda familiar (salário mínimo) } \\
\hline$<1$ & 31 & 12,9 \\
\hline $1-3$ & 63 & 26,2 \\
\hline $3-5$ & 34 & 14,2 \\
\hline $5-7$ & 34 & 14,2 \\
\hline $7-9$ & 22 & 9,2 \\
\hline$>9$ & 09 & 3,7 \\
\hline Não informou & 47 & 19,6 \\
\hline Total & 240 & 100,0 \\
\hline \multicolumn{3}{|l|}{ Saber ler e escrever } \\
\hline Sim & 192 & 80,0 \\
\hline Não & 48 & 20,0 \\
\hline Total & 240 & 100,0 \\
\hline \multicolumn{3}{|l|}{ Escolaridade (anos) } \\
\hline 0 a 5 & 114 & 47,5 \\
\hline 6 a 10 & 59 & 24,6 \\
\hline 11 a 15 & 40 & 16,7 \\
\hline $15 \mathrm{e}+$ & 27 & 11,2 \\
\hline Total & 240 & 100,0 \\
\hline
\end{tabular}

Fonte: Dados da pesquisa direta realizada em João Pessoa-PB.

No aspecto renda familiar, observa-se que a maioria $(26,2 \%)$ dos idosos possui uma renda variando de 1 a 3 salários mínimos, consideran- 
do o salário vigente à época da pesquisa de $\mathrm{R} \$$ 510,00 (quinhentos e dez reais). Nesse sentido, estudos realizados por Lebrão e Laurenti ${ }^{16}$, revelaram que a maioria dos idosos recebia em média 2,1 salários mínimos e são os provedores da família, dividindo-o para atender as despesas da sua casa, tais como: alimentação, moradia, transporte, entre outros.

Com relação ao fator escolaridade, os dados revelam que $80 \%$ dos participantes informaram que sabiam ler e escrever e os $20 \%$ restantes disseram que não, dentre estes, $47,5 \%$ frequentaram a escola por um período mínimo de 5 anos. Tal fato merece especial atenção/reflexão haja vista retratar-se de um quadro social e de saúde crucial. Diante do exposto, observa-se a necessidade de políticas públicas do Ministério da Educação e Cultura (MEC) voltadas para a inclusão dos idosos no sistema educacional de ensino, principalmente para aqueles que deixaram de estudar ou não foram devidamente alfabetizados. Com essa finalidade, programas como da Educação de Jovens e Adultos (EJA) e Brasil Alfabetizado (PBA) foram criados e implementados por tal ministério ${ }^{17}$.

Entretanto, essa iniciativa nos chama a atenção para o fato do governo brasileiro não estabelecer um programa de educação específico e direcionado para a pessoa idosa, em respaldo à garantia do seu direito à educação, respeitando a peculiaridade de idade estabelecida no Estatuto do Idoso $^{18}$.

Na Tabela 2, em anexo, estão listados os dados que relacionam a influência das variáveis sociodemográficas com depressão, segundo a classificação da EDG (Escala de Depressão Geriátrica). Desses dados verifica-se que dos 240 idosos, $75,8 \%$ foram considerados como normais; $19,6 \%$ com depressão de grau leve e apenas 4,6\% com depressão severa. Esses resultados, de certa forma já eram esperados, mesmo porque, no Brasil, a presença da depressão entre os idosos vem aumentando significativamente nos últimos anos, com uma prevalência variando nesse grupo de $4,7 \%$ a $36,8 \%$, sendo considerado como um dos problemas psiquiátricos mais comuns encontrados nas pessoas idosas ${ }^{3}$.

A partir da análise da Tabela 2 também pôde se observar que há clara significância na associação entre as variáveis sociodemográficas e os níveis de depressão apresentados pelos participantes. Esses dados tornam-se importantes, pois demonstram que os níveis de classificação da depressão podem atingir, de forma desigual, no que se refere ao gênero e ao saber ler e escrever, por apresentar um Qui-quadrado de 13,3 e 10,468, segundo a análise pelo software SPSS.

Em relação ao gênero, verificou-se uma predominância do quadro depressivo feminino, quando 29,9\% das 167 mulheres do estudo apresentaram uma classificação de depressão de grau leve ou severo. Nesse caso, talvez o fato da mulher, em relação ao homem, ao enfrentar a viuvez ser menos propensa a buscar uma nova união, seja um fator que contribua para uma maior predisposição a essa patologia. Estudos indicam que a viuvez é enfrentada de forma diferente entre os gêneros feminino e masculino, uma vez que o primeiro tende a permanecer só, enquanto que o segundo, em geral, casa-se novamente ${ }^{19}$.

Estudos confirmam que, em relação ao gênero, a depressão quando diagnosticada nos indivíduos com idade igual ou superior a 60 anos, é mais prevalente nas mulheres do que nos homens, ou seja, são 02 (duas) mulheres acometidas para cada homem ${ }^{3}$. Porém, no presente estudo, ao aplicar a proporcionalidade em relação à depressão entre ambos os gêneros (50 mulheres e 08 homens), constatou-se que, para cada homem, aproximadamente, 6,3 mulheres apresentam algum quadro depressivo, confirmando uma maior prevalência de sintomas da depressão no sexo feminino.

Quanto à idade dos participantes, observouse uma maior frequência da sintomatologia depressiva na faixa etária entre 71 a 76 anos, com $7,5 \%$ dos idosos. Ainda que a maioria dessa população possa ser considerada saudável psiquicamente, eles são muito mais vulneráveis aos distúrbios psiquiátricos do que as pessoas mais jovens. A depressão é mais comumente presente a partir dos 70 anos, pois a sua prevalência aumenta significativamente após os 75 anos de idade. Nas pessoas que ainda não chegaram aos 60 anos, mas que estão próximas da aposentadoria, a prevalência dessa morbidade tende a aumentar nos anos que a antecedem, diminuindo na década subsequente, e torna a se elevar após os 70 anos $^{3}$.

Com relação ao estado civil, pôde-se observar que, dos 126 idosos casados, 81,0\% não apresentaram sintomatologia depressiva. Porém, $16,0 \%$ deles apresentaram no grau leve e 3,0\% severa. Entre os viúvos, o quadro depressivo foi mais evidenciado, uma vez que de $79,20,3 \%$ apresentaram depressão de grau leve e 5,1\% com o grau severo. Os dados demonstram uma diferença significativa entre os idosos que residem com um companheiro/casados (126 idosos), em relação àqueles que residiam sozinhos (viúvos). Essa realidade identificada ergue a hipótese so- 
Tabela 2. Distribuição das variáveis sociodemográficas dos idosos em relação ao grau de depressão - João Pessoa (PB), 2011.

\begin{tabular}{|c|c|c|c|c|c|c|c|c|}
\hline \multirow{3}{*}{ Variável } & \multicolumn{8}{|c|}{ Grau de depressão } \\
\hline & \multicolumn{2}{|c|}{ Normal } & \multicolumn{2}{|c|}{ Leve } & \multicolumn{2}{|c|}{ Severa } & \multicolumn{2}{|c|}{ Total } \\
\hline & $\mathrm{N}$ & $\%$ & $\mathrm{~N}$ & $\%$ & $\mathrm{~N}$ & $\%$ & $\mathrm{~N}$ & $\%$ \\
\hline \multicolumn{9}{|l|}{ Idade (anos) } \\
\hline 60 a 65 & 47 & 25,8 & 09 & 19,1 & 02 & 18,2 & 58 & 24,2 \\
\hline 66 a 70 & 45 & 24,7 & 12 & 25,5 & 01 & 9,1 & 58 & 24,2 \\
\hline 71 a 76 & 34 & 18,7 & 14 & 29,8 & 04 & 36,3 & 52 & 21,6 \\
\hline 77 a 80 & 33 & 18,1 & 06 & 12,8 & 02 & 18,2 & 41 & 17,1 \\
\hline 81 a 85 & 18 & 10,0 & 03 & 6,4 & - & - & 21 & 8,7 \\
\hline$>86$ & 05 & 2,7 & 03 & 6,4 & 02 & 18,2 & 10 & 4,2 \\
\hline Total & 182 & 100,0 & 47 & 100,0 & 11 & 100,0 & 240 & 100,0 \\
\hline \multicolumn{9}{|l|}{ Sexo } \\
\hline Masculino & 65 & 35,7 & 04 & 8,5 & 04 & 36,4 & 73 & 30,4 \\
\hline Feminino & 117 & 64,3 & 43 & 91,5 & 07 & 63,6 & 167 & 69,6 \\
\hline Total & 182 & 100,0 & 47 & 100,0 & 11 & 100,0 & 240 & 100,0 \\
\hline \multicolumn{9}{|l|}{ Estado civil } \\
\hline Solteiro (a) & 14 & 7,7 & 08 & 17,0 & 01 & 9,0 & 23 & 9,6 \\
\hline Casado (a) & 102 & 56,0 & 20 & 42,6 & 04 & 36,5 & 126 & 52,5 \\
\hline Divorciado (a) & 05 & 2,8 & - & - & 01 & 9,0 & 06 & 2,5 \\
\hline Separado (a) & 01 & 0,5 & 03 & 6,4 & 01 & 9,0 & 05 & 2,1 \\
\hline Viúvo (a) & 59 & 32,5 & 16 & 34,0 & 04 & 36,5 & 79 & 32,9 \\
\hline Outros (NS/NR) & 01 & 0,5 & - & - & - & - & 01 & 0,4 \\
\hline Total & 182 & 100,0 & 47 & 100,0 & 11 & 100,0 & 240 & 100,0 \\
\hline \multicolumn{9}{|l|}{ Religião } \\
\hline Nenhuma & 02 & 1,27 & 01 & 2,1 & 01 & 9,1 & 04 & 1,7 \\
\hline Católica & 132 & 2,52 & 33 & 70,2 & 08 & 72,7 & 173 & 72,1 \\
\hline Protestante & 42 & 3,1 & 10 & 21,3 & 02 & 18,2 & 54 & 22,5 \\
\hline Espírita & - & - & 01 & 2,1 & - & - & 01 & 0,4 \\
\hline Judaica & 01 & 0,5 & - & - & - & - & 01 & 0,4 \\
\hline Outra religião & 05 & 2,7 & 02 & 4,3 & - & - & 07 & 2,9 \\
\hline Total & 182 & 100,0 & 47 & 100,0 & 11 & 100,0 & 240 & 100,0 \\
\hline \multicolumn{9}{|l|}{ Renda } \\
\hline$<1$ & 16 & 8,8 & 14 & 29,8 & 01 & 9,1 & 31 & 12,9 \\
\hline $1-3$ & 49 & 26,9 & 12 & 25,5 & 02 & 18,2 & 63 & 26,2 \\
\hline $3-5$ & 31 & 17,0 & 02 & 4,3 & 01 & 9,1 & 34 & 14,2 \\
\hline $5-7$ & 28 & 15,4 & 03 & 6,4 & 03 & 27,2 & 34 & 14,2 \\
\hline $7-9$ & 16 & 8,8 & 04 & 8,5 & 02 & 18,2 & 22 & 9,2 \\
\hline$>9$ & 04 & 2,2 & 04 & 8,5 & 01 & 9,1 & 09 & 3,7 \\
\hline Não informou & 38 & 20,9 & 08 & 17,0 & 01 & 9,1 & 47 & 19,6 \\
\hline Total & 182 & 100,0 & 47 & 100,0 & 11 & 100,0 & 240 & 100,0 \\
\hline \multicolumn{9}{|l|}{ Saber ler e escrever } \\
\hline Sim & 154 & 84,6 & 30 & 63,8 & 08 & 72,7 & 192 & 80,0 \\
\hline Não & 28 & 15,4 & 17 & 36,2 & 03 & 27,3 & 48 & 20,0 \\
\hline Total & 182 & 100,0 & 47 & 100,0 & 11 & 100,0 & 240 & 100,0 \\
\hline \multicolumn{9}{|l|}{ Escolaridade (anos) } \\
\hline 0 a 5 & 82 & 45,1 & 27 & 57,4 & 05 & 45,4 & 114 & 47,5 \\
\hline 6 a 10 & 45 & 24,7 & 11 & 23,5 & 03 & 27,3 & 59 & 24,6 \\
\hline 11 a 15 & 29 & 15,9 & 08 & 17,0 & 03 & 27,3 & 40 & 16,7 \\
\hline $15 \mathrm{e}+$ & 26 & 14,3 & 01 & 2,1 & - & - & 27 & 11,2 \\
\hline Total & 182 & 100,0 & 47 & 100,0 & 11 & 100,0 & 240 & 100,0 \\
\hline
\end{tabular}


bre os idosos que vivem só possam referenciar, com maior frequência, sintomas depressivos.

No aspecto de morar só e/ou não ter um companheiro (a) poderá contribuir a presença de um quadro depressivo, que pode estar associado ao processo de solidão vivenciado pelo idoso. Os outros fatores que contribuem para o surgimento de sintomas depressivos em um indivíduo são as causas externas, como a perda de entes queridos e a perda de status social ${ }^{20,21}$.

Em relação à religião, os dados indicam que os idosos, sem qualquer tipo de religião, apresentaram grau depressivo que merece atenção, quando dos 04 idosos, 50\% desses demonstraram grau de depressão leve ou severo.

A realidade da classificação da depressão encontrada entre esses idosos, quando nos referimos à situação de não vivenciarem religião, explica-se pelo fato de poderem estar associado a um processo de solidão. A partir do momento em que os idosos participam, ou não, de algum grupo ou atividades religiosas que são oferecidas à comunidade, podem contribuir para o não aparecimento de sintomas depressivos e para uma melhor qualidade de vida. Segundo estudo realizado Paradela et al..$^{20}$ a solidão pode ser um fator determinante para o aparecimento de quadro depressivo.

Quanto à renda familiar, associada ao grau de depressão, verificou-se que, dos 240 idosos, os que recebem menos de um salário, os que recebem mais de nove salários mínimos ou que não informaram a renda, constituindo-se a maioria (182 idosos), não apresentaram grau de depressão. Dos 47 idosos que se encontram com grau de classificação leve, destacam-se aqueles que recebem menos de um salário e até três salários mínimos, ou seja, 29 idosos. Entre os 11 idosos com grau de depressão severa, os dados nos chamam atenção para os 03 (três), 27,2\%, que recebem de 05 (cinco) a 07 (sete) salários mínimos.

Um dado relevante foi a presença de sintomatologia depressiva entre o total de 09 idosos que informaram ter renda familiar superior a 09 salários mínimos, 04 (44,4\%) se apresentam com grau de depressão leve e $01(11,2 \%)$ com grau de depressão severa.

No que diz respeito ao nível de escolaridade, verificou-se que, do total de 182 idosos que não apresentaram grau de depressão, 84,6\% sabem ler e escrever, e 15,4\% não possuem essa qualificação. Porém, dos 58 idosos que apresentam grau de depressão, a maioria, que se destaca no grau leve $(63,8 \%)$ e severo $(72,7 \%)$, sabem ler e escrever. Quando considerado o nível de escolaridade, em anos estudados, associado ao grau de depressão, um total de $32(13,3 \%)$ com zero a 05 anos de estudo possuem sintomatologia depressiva, sendo $84,4 \%$ (27 idosos) com depressão de grau leve e 15,6\% (05 idosos) com depressão severa.

No Brasil, em relação ao analfabetismo, os idosos que estudaram no máximo 03 (três) anos, e têm idade de 75 anos ou mais, foram considerados analfabetos funcionais. Porém, a proporção de idosos que completaram pelo menos o ensino médio subiu de 7,5\% para 10,5\%, ou seja, $40 \%$ de aumento. As pesquisas apontam que 59,4\% dos idosos são responsáveis pelo domicílio ${ }^{22}$.

Segundo Maciel e Guerra ${ }^{14}$ o grande número de pessoas analfabetas em nosso país ratifica a idéia de uma rede de serviços sociais ineficiente durante a vida. Tal ineficiência pode ser observada ainda em outros aspectos, tais como: habitação, cultura, renda e saúde. O que preocupa é que a saúde mantém íntima relação com a escolaridade. Relacionado a isso, ressalta-se o fato de as pessoas menos favorecidas, em termos educacionais, não terem maiores preocupações com sua saúde e capacidade de recuperação, além da tendência a possuírem hábitos higiênicos pouco saudáveis.

\section{Considerações finais}

Este estudo procurou avaliar a sintomatologia da depressão autorreferida por idosos em João Pessoa (PB), em que se observou um grau mais significativo da classificação da sintomatologia da depressão (leve e severa). Entre os idosos participantes do estudo, comparando às características sociodemográficas, observa-se que a maioria deles se encontrava na faixa etária entre 71 e 76 anos, predominando o gênero feminino, nos casados ou viúvos, com religião, renda familiar variando de menos de 1 a 3 salários mínimos, que sabem ler e escrever, e com menos de 05 anos de estudo. Outro dado significativo refere-se ao fato de que idosos com um maior grau de escolaridade e maior renda familiar apresentarem sintomatologia depressiva compatível com grau severo.

Dessa forma, salienta-se que o aparecimento da sintomatologia da depressão em idosos poderá estar relacionado às condições socioeconômicas, culturais e aos aspectos biológicos, em especial dos participantes deste estudo.

Tal situação ratifica a necessidade da elaboração de políticas públicas de saúde, bem como da sua efetiva implantação e implementação, para dirimir as desigualdades socioculturais que se 
apresentam no grupo, com a finalidade de viabilizar e facilitar os benefícios oferecidos aos idosos, e que, certamente, possam garantir um atendimento integral e de qualidade, capaz de minimizar os riscos à sua saúde. Nesse enfoque, o alcance do envelhecimento ativo, referenciado pela Organização Mundial da Saúde, tanto para países desenvolvidos quanto para países em desenvolvimento, teria uma possibilidade de concretização.

\section{Colaboradores}

MF Oliveira, VP Bezerra e AO Silva participaram da concepção, delineamento, análise e interpretação dos dados, redação do artigo e revisão crítica aprovação da versão a ser publicada; MSCF Alves, MASP Moreira e CP Caldas da redação do artigo, revisão crítica e aprovação da versão a ser publicada.

\section{Referências}

1. World Health Organization (WHO). Envelhecimento ativo: uma politica de saúde. Trad. Suzana Gontijo. Brasília: Organização Pan-Americana da Saúde; 2005.

2. Ohara ECC, Ribeiro MP. Saúde do idoso. In.: Ohara ECC, Saito RXS, organizadores. Saúde da família: considerações teóricas e aplicabilidade. São Paulo: Martinari; 2008.

3. Brasil. Ministério da Saúde (MS). Secretaria de Atenção à Saúde. Departamento de Atenção Básica. Envelhecimento e saúde da pessoa idosa. $1^{\text {a }}$ ed. Brasília: MS; 2007.

4. Sadock BJ, Sadock VA. Compêndio de psiquiatria: ciências do comportamento e psiquiatria clínica. Tradução de Cláudia Oliveira Dornelles, Cristina Monteiro, Irineo S. Ortiz e Ronaldo Cataldo Costa. $9^{\mathrm{a}}$ ed. Porto Alegre: Artmed; 2007.

5. Hales RE. Tratado de psiquiatria clínica. Trad. Cláudia Dornelles, Cristina Monteiro e Ronaldo Cataldo Costa. 4a ed. Porto Alegre: Artmed; 2006.

6. Wharton RN. Transtornos afetivos. In. Rowland LP, Pedley TA, organizadores. Tratado de neurologia. Tradução de Fernando Diniz Mundim. $11^{\text {a }}$ ed. Rio de Janeiro: Guanabara Koogan; 2007.

7. Smeltzer SC, Bare BG, Hinkle JL, Cheever KH. Tratado de enfermagem médico-cirúrgica. $11^{\text {a }}$ ed. Rio de Janeiro: Guanabara; 2008.

8. Felten BS, Gray-Vickrey P, Mangin EJ, Purvis G, Ross-Kerr JC, Vontz MJ. Geriatria e gerontologia. Tradução de Carlos Henrique Consendey. Rio de Janeiro: Reichmann \& Autores Editores; 2005.

9. Dalgalarrondo P. Psicopatologia e semiologia dos transtornos mentais. 2a ed. Porto Alegre: Artmed; 2008.

10. Nunes Filho EP, Bueno JR, Nardi AE. Psiquiatria e saúde mental: conceitos clínicos e terapêuticos fundamentais. São Paulo: Atheneu; 2005.

11. Instituto Brasileiro de Estatística e Geografia (IBGE). Contagem da população 2007. [site na Internet]. [acessado em 2011 mar 23]. Disponível em: http://www. ibge.gov.br.

12. Brasil. Ministério da Saúde (MS). Conselho Nacional de Saúde. Resolução 196/96. Diretrizes e normas regulamentadoras em pesquisa envolvendo seres humanos. Brasília: MS; 1996.
13. Neri AL. Palavras-chave em gerontologia. $3^{\mathrm{a}}$ ed. Campinas: Alínea; 2008.

14. Maciel ÁCC, Guerra RO. Influência dos fatores biopsicossociais sobre a capacidade funcional de idosos residentes no nordeste do Brasil. Rev. Bras. de Epidemiol. 2007; 10(2):178-189.

15. Wikipédia. Catolicismo no Brasil. [site da Internet]. [acessado em 2012 jul 15]; Disponível em: http:// pt.wikipedia.org/wiki/Catolicismo_romano _no_Brasil

16. Lebrão ML, Laurenti R. Saúde, bem-estar e envelhecimento: o estudo SABE no município de São Paulo. Rev. Bras. de Epidemiol. 2005; 8(2):127-141.

17. Brasil. Ministério da educação e cultura. [acessado em 2011 mar 23]. Disponível em: http://portal.mec. gov.br/index.php

18. Brasil. Lei no 10.741, de 10 de outubro de 2003. Dispõe sobre o Estatuto do Idoso e dá outras providências. Diário Oficial da União 2003; 10 out.

19. Paz AA, Santos BRL, Eidt OR. Vulnerabilidade e envelhecimento no contexto da saúde. Acta paul. Enferm 2006;19(3):338-342

20. Paradela EMP, Lourenço RA, Veras RP. Validação da escala de depressão geriátrica em um ambulatório geral. Rev Saude Publica 2005; 39(6): 918-923.

21. Zimerman GI. Velhice: aspectos biopsicossociais. Porto Alegre: Artmed; 2000.

22. Instituto Brasileiro de Estatística e Geografia (IBGE). Censo populacional de 2010. [documento da Internet\}. [acessado em 2012 jul 12]; Disponível em: http:// www.ibge.gov.br/home/estatistica/populacao/censo2010/calendario.shtm.

Artigo apresentado em 19/11/2011

Aprovado em 18/01/2012

Versão final apresentada em 02/02/2012 